\title{
On the synchronization of logistic maps
}

\author{
Ömer Morgül ${ }^{1}$ \\ Department of Electrical and Electronics Engineering, Bilkent University, 06533 Bilkent, Ankara, Turkey
}

Received 9 July 1997; revised manuscript received 11 March 1998; accepted for publication 29 July 1998

Communicated by A.P. Fordy

\begin{abstract}
We study the synchronization of two chaotic maps described by a logistic equation. We propose a new feedback term and show that the resulting system has some desired properties including exponential synchronization with arbitrary decay rate, and robustness with respect to parameter mismatch and noise. We also present some simulation results and some possible extensions of this scheme to the synchronization of other chaotic systems. (c) 1998 Elsevier Science B.V.
\end{abstract}

Keywords: Synchronization; Chaotic systems; Logistic map

\section{Introduction}

In recent years, the synchronization of chaotic systems has received a great deal of attention from scientists from various disciplines, see, e.g., Refs. [1,2]. It has been shown that two identical chaotic systems can synchronize when a suitable feedback (or coupling) is introduced in one (or both) of the systems $[3,4]$. One of the motivations for synchronization is the possibility of sending messages through chaotic systems for secure communication $[5,6]$. In such applications, the message signal is transmitted to the receiver by using a chaotic signal as a carrier, and synchronization is required at the receiver to recover the message signal $[7,8]$. There are some methods which could be used for synchronization $[9,10]$, and one such method is known as the variable feedback method [11]. A related problem encountered in the feedback control systems is the problem of state estimation. Assume that a system, whose states are not available from mea-

\footnotetext{
${ }^{1}$ E-mail: morgul@ee.bilkent.edu.tr.
}

surements, is given. The state estimation problem is to establish another system, called an observer, which provides an estimation for the states of the original system. The states of the observer then could be used to design a feedback control law to control the original system, see, e.g., Ref. [12]. The idea of observer design could naturally be used for synchronization of two chaotic systems $[10,13]$.

In a recent work [14], a variable feedback approach for the synchronization of logistic maps was considered and it was shown that, with the choice of the feedback laws given in Ref. [14], the synchronization property does not change if both the drive and the response systems are subjected to a common noise. In this paper we propose a similar synchronization scheme which enjoys the same properties along with some other ones which may not hold for the feedback laws proposed in Ref. [14].

In this report, we apply the variable feedback method to the synchronization of two logistic maps. The coupled systems, including the coupled logistic equations, have been investigated by many re- 
searchers. In Ref. [15], the synchronization property of coupled maps, including the tent maps, were investigated. In Ref. [16], a different type of coupling for logistic maps and its various properties were investigated. In Ref. [17], a new approach to determine the existence of synchronized states for some chaotic systems was proposed. In Ref. [18], coupled tent maps and their various synchronization properties were given.

\section{Synchronization scheme}

We consider the systems given by the following equations in discrete time,

$x_{n+1}=4 x_{n}\left(1-x_{n}\right)$.

$y_{n+1}=4 y_{n}\left(1-y_{n}\right)+G\left(x_{n}, y_{n}\right)$,

where $n=0,1, \ldots$ is the discrete time and $G(\cdot, \cdot)$ is the variable feedback. Here (1) and (2) represent the drive and the response systems respectively. The aim is to choose the term $G(\cdot, \cdot)$ such that for any initial conditions $x_{0}$ and $y_{0}$, we have $\lim _{n \rightarrow \infty}\left|x_{n}-y_{n}\right|=0$; moreover, for practical purposes it is preferable if we can achieve any decay rate.

The choice of $G(\cdot, \cdot)$ is not unique and as is stated in Ref. [14], in most cases it could be found by a trial and error procedure. In Ref. [14], $G\left(x_{n}, y_{n}\right)=\left(y_{n}-\right.$ $\left.x_{n}\right)\left(2 x_{n}-1\right)$ and $G\left(x_{n}, y_{n}\right)=\left(y_{n}-x_{n}\right)\left(2 x_{n}-1\right)^{3}$ were considered and it was claimed that with these choices the synchronization property does not change when both (1) and (2) are subjected to common noise. The analysis given in Ref. [14] was based on extensive simulation. In this report we propose a new choice for $G(\cdot,$,$) which has the following advantages;$

(i) the synchronization error decays exponentially to zero and the decay rate can be assigned arbitrarily;

(ii) if both the drive and the response systems are subjected to common noise, the synchronization property stated above still holds;

(iii) the proposed scheme is robust with respect to noise and parameter mismatch;

(iv) the proposed scheme could be used to generate some other synchronizing feedback laws, for which the property (ii) holds ( this gives a partial justification for a conjecture made in Ref. [14]);

(v) the analysis could be done rigorously.

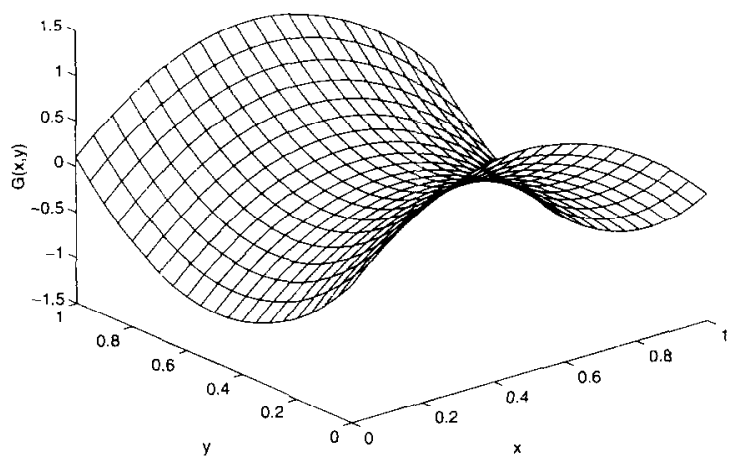

Fig. 1. $G(x, y)$ versus $x$ and $y$ for $\rho=0.1$.

We propose the following feedback law,

$G\left(x_{n}, y_{n}\right)=\left[4\left(1-x_{n}-y_{n}\right)-\rho\right]\left(x_{n}-y_{n}\right)$,

where $\rho$ is an arbitrary real number satisfying $|\rho|<1$. A three-dimensional figure of $G(x, y)$ versus $x$ and $y$ for $\rho=0.1$ is shown in Fig. 1. To analyze (1)-(3), we define the following error term,

$e_{n}=x_{n}-y_{n}$.

By using (1)-(3), we obtain the following which describes the error dynamics,

$e_{n+1}=\rho e_{n}$,

hence the error can be computed as

$e_{n}=\rho^{n} e_{0}$.

Obviously, since $|\rho|<1$, the error decays exponentially to zero and by choosing $\rho$ appropriately, we could achieve arbitrary decay rates. For clarity, we will state this property as follows. Note that, since $0 \leqslant$ $x_{n} \leqslant 1$ in the chaotic regime, without loss of generality, we may assume that $|e(0)| \leqslant 1$. The following fact proves the property (i) stated above.

Fact 1. Consider the system given by (1)-(3).

(a) If $|\rho|<1$, then for any $e_{0}$ the error decays exponentially to zero.

(b) Assume $\left|e_{0}\right| \leqslant 1$ and let $p$ and $N$ be two given integers and choose $\rho=10^{-\delta}$ for some $\delta>0$. If $\delta \geqslant$ $(p+1) / N$, then the first $p+1$ digits of $e_{n}$ will be 0 , $\forall n \geqslant N$.

Proof. The first part is obvious from (6). To prove the second part, note that if $\left|e_{0}\right| \leqslant 1$, then from (6) 
we see that $\left|e_{n}\right| \leqslant|\rho|^{n}$. Then for any given $\epsilon>0$, we see that $\left|e_{n}\right| \leqslant \epsilon$ for $n \geqslant \log \epsilon / \log |\rho|$. The first $p+1$ digits of $e_{n}$ will be 0 for $n \geqslant N$ if $\left|e_{n}\right| \leqslant 10^{-(p+1)}$ for $n \geqslant N$. Hence, by choosing $\epsilon=10^{-(p+1)}$, we see that the stated property holds for $n \geqslant \log \epsilon / \log |\rho|=$ $(p+1) / \delta$, hence for $\delta \geqslant(p+1) / N$.

Remark 1. At this point we compare the exponential synchronization property given above with the concept of monotone synchronization given in Ref. [19]. Let us assume that the systems to be synchronized are given in $\mathbb{R}^{n}, n \geqslant 1$, and let $\|\cdot\|$ be any norm in $\mathbb{R}^{n}$. If the synchronization crror $\left\|e_{k}\right\|$ monotonically decreases to 0 , we say that the synchronization is monotone. If there exist constants $M>0$ and $|\rho|<1$ such that the following holds,

$$
\left\|e_{k}\right\| \leqslant M|\rho|^{k}\|e(0)\|,
$$

then we say that the synchronization is exponential.

(i) In both cases, $e=0$ is an asymptotically stable equilibrium for the error dynamics.

(ii) Exponential synchronization may or may not be monotone, and vice versa. For our example, the error given by (6) decays both exponentially and monotonically. But one can easily construct examples such that one type of synchronization property holds but the other does not.

(iii) It is obvious that the monotone synchronization property depends on the norm used to evaluate $\left\|e_{k}\right\|$. However, the exponential synchronization property is independent of the norm used in $\mathbb{R}^{n}$. This is due to the fact that all norms in $\mathbb{R}^{n}$ are equivalent. To see this, let $\|\cdot\|_{a}$ be a norm in $\mathbb{R}^{n}$ for which (7) holds, and let $\|\cdot\|_{b}$ denote any other norm in $\mathbb{R}^{n}$. Because of the norm equivalence in $\mathbb{R}^{n}$, there exist constants $c_{1}>0$ and $c_{2}>0$ such that the following holds,

$c_{1}\|e\|_{b} \leqslant\|e\|_{a} \leqslant c_{2}\|e\|_{b}$.

By using (7) and (8), we obtain the following,

$\left\|e_{k}\right\|_{b} \leqslant \frac{c_{2} M}{c_{1}}|\rho|^{k}\left\|e_{0}\right\|_{b}$,

i.e. with respect to norm $\|\cdot\|_{b}$ the synchronization is still exponential.

Now we assume that the drive and response systems given by (1) and (2) are subjected to noise as follows, $x_{n+1}=4 x_{n}\left(1-x_{n}\right)+\nu_{n}$,

$y_{n+1}=4 y_{n}\left(1-y_{n}\right)+G\left(x_{n}, y_{n}\right)+\tilde{\nu_{n}}$,

where $\nu_{n}$ and $\tilde{\nu_{n}}$ represent noise terms. These terms are assumed to be bounded, but arbitrary otherwise. With the feedback term (3), we have the following, which proves the property (ii) stated above and more.

Fact 2. Consider the systems given by (10), (11) and (3), where $\nu_{n}$ and $\tilde{\nu_{n}}$ are bounded but arbitrary otherwise.

(a) If $\nu_{n} \equiv \tilde{\nu_{n}}$, then the conclusions of Fact 1 hold.

(b) If $\nu_{n} \neq \tilde{\nu_{n}}$, then the synchronization error remains bounded. Moreover, this bound depends linearly on $\nu_{\max }=\sup _{n}\left|\nu_{n}-\tilde{\nu_{n}}\right|$.

Proof. By defining the error as in (4), and using (10) and (11), we obtain the following error dynamics,

$e_{n+1}=\rho e_{n}+\nu_{n}-\tilde{\nu_{n}}$.

If $\nu_{n} \equiv \tilde{\nu}_{n}$, then (12) reduces to (5), hence (6) and consequently Fact 1 holds. For the case $\nu_{n} \neq \tilde{\nu}_{n}$, we obtain the following,

$\left|e_{n+1}\right| \leqslant|\rho|\left|e_{n}\right|+\nu_{\max }$.

Hence we obtain

$$
\begin{aligned}
& \left|e_{n}\right| \leqslant|\rho|^{n}|e(0)|+\nu_{\max } \sum_{i=0}^{n-1} \rho^{i} \leqslant|\rho|^{n}|e(0)| \\
& \quad+\nu_{\max } /(1-\rho),
\end{aligned}
$$

which proves part (b). If we define $e_{\infty}$ by $e_{\infty}=$ $\nu_{\max } /(1-\rho)$, and the set $S_{\infty}$ as the closed interval $\left[-e_{\infty}, e_{\infty}\right]$, then (14) implies that $e_{n} \rightarrow S_{\infty}$ as $n \rightarrow$ $\infty$.

We note that Fact 2 holds even if the solutions of (10) and (11) are unbounded. Even if $\nu_{\max }$ is large, hence $e_{\infty}$ is large, the error remains bounded, i.e. such bounded noises cannot destabilize the error. In other words, this scheme is robust with respect to noise. Also in the case of common noise, for the choices of $G\left(x_{n}, y_{n}\right)$ proposed in Ref. [14] the transition times to decrease the first $p$ digits of the error to zero may depend on the noise, whereas in our case a uniform 
transition time, independent of noise, could be obtained, see Fact 1 . Note that case (b) of Fact 2 was not considered in Ref. [14].

Next we will prove that a result similar to Fact 2 holds when the parameters of the drive and response systems do not match. We assume that the drive system is given by (10) and the response system is given by the following,

$$
y_{n+1}=4 y_{n}\left(1-y_{n}\right)+G\left(x_{n}, y_{n}\right)+\tilde{\nu_{n}}+\Delta y_{n}\left(1-y_{n}\right),
$$

where $\Delta$ is a real number representing the parameter mismatch between the drive and the response systems. The following fact proves the property (iii) stated above.

Fact 3. Consider the systems given by (10), (15) and (3). Assume that the noise terms $\nu_{n}, \tilde{\nu_{n}}$ and $\Delta$ are such that the solutions of (10) and (15) are bounded. Let us define $\nu_{\max }$ as in Fact 2 and $Y_{m}=\sup _{n} \mid y_{n}(1-$ $\left.y_{n}\right) \mid$. Then the synchronization error remains bounded and this bound depends linearly on $\Delta$ and $\nu_{\max }$.

Proof. In this case, the error dynamics becomes

$e_{n+1}=\rho e_{n}+\nu_{n}-\tilde{\nu_{n}}-\Delta y_{n}\left(1-y_{n}\right)$.

Hence, we obtain

$\left|e_{n+1}\right| \leqslant|\rho|\left|e_{n}\right|+\nu_{\max }+|\Delta| Y_{m}$.

Therefore, similar to (14), we obtain

$\left|e_{n}\right| \leqslant|\rho|^{n}|e(0)|+\left(Y_{m}|\Delta|+\nu_{\max }\right) /(1-\rho)$.

Remark 2. We note that by Facts 2 and 3, the synchronization scheme proposed here is robust with respect to noise and parameter mismatch, and this point is quite important from a practical point of view. Similar to the proof of the Fact 2, let $e_{\infty}$ denote $e_{\infty}=$ $\left(Y_{m}|\Delta|+\nu_{\max }\right) /(1-\rho)$ and let $S_{\infty}$ denote the set $\left[-e_{\infty}, e_{\infty}\right]$. According to Fact 3 , in the presence of bounded noise and parameter mismatch, the synchronization error asymptotically approaches to $S_{\infty}$, hence remains bounded. The error bound $e_{\infty}$ given above may be large, but it shows the boundedness of the

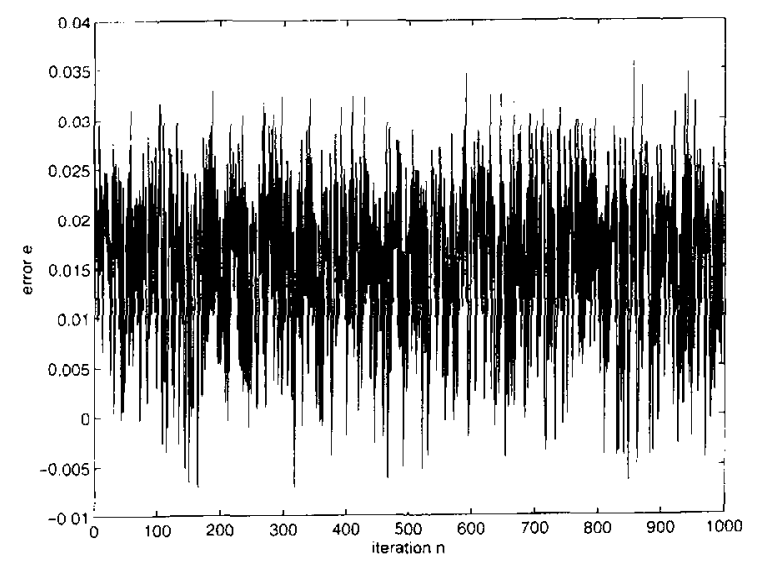

Fig. 2. Error signal (plotted for $n \geqslant 5$ )

error. hence such bounded noise and parameter mismatch cannot destabilize the error under the conditions of Fact 3. Moreover, this bound depends linearly on $\nu_{\max }$ and $|\Delta|$, hence the error decreases as these terms decrease. We note that this robustness result is a consequence of exponential synchronization, see also Ref. $[10,13]$.

We also simulated (10) and (15) for $\Delta=-0.1$ and $\rho=0.1$. Noise terms $\nu$ and $\tilde{\nu}$ are generated randomly by the computer, which are uniformly distributed in $[0,0.01]$. At each step, similar to Ref. [14], we considered only the values of $\nu_{n}$ yielding $0 \leqslant x_{n+1} \leqslant 1$. For initial conditions we chose $x_{0}=0.1, y_{0}=0.9$. The corresponding error in 1000 iterations are shown in Fig. 2. In this figure we plot $e_{n}$ for $n \geqslant 5$ in order to eliminate the transients due to large initial error. $\Lambda \mathrm{f}$ ter the simulations we observed that $\nu_{\max }=0.009437$, $Y_{m}=0.249991$, and since $|\Delta|=0.1$, we computed the error bound $e_{\infty}$ given in Remark 2 as $e_{\infty}=0.038262$. On the other hand, according to simulation results, the maximum error is (for $n \geqslant 5$ ) $e_{m}=0.035728$. Hence the error bound given in Remark 2 is quite close to the actual error bound. For the cases where $\Delta$ and/or the noise bound is small we obtained smaller errors.

\section{Possible generalizations}

The synchronization scheme presented above could be generalized to obtain a class of synchronizing feedback laws as follows. Suppose that for some function 
$f$, the following error dynamics is known to be asymptotically stable,

$e_{n+1}=f\left(e_{n}\right)$,

i.e. for any $e_{0}, \lim _{n \rightarrow \infty} e_{n}=0$. Note that for the logistic equation it is sufficient that this property holds for $\left|e_{0}\right|<1$. If we choose the feedback law as

$G\left(x_{n}, y_{n}\right)=4\left(1-x_{n}-y_{n}\right) e_{n}-f\left(e_{n}\right)$,

then (1), (2) and (20) will result in the error dynamics given by (19), hence synchronization can be achieved. Moreover, in case of common noise, since these terms cancel each other in the error equation, the proposed scheme will retain its synchronization property. This proves that the conjecture made at the end of Ref. [14] holds for the class of synchronizing feedback laws given by (20). However, for an arbitrary asymptotically stable dynamics, exponential synchronication may not hold, and consequently part (b) of Fact 2 and Fact 3 may not hold.

Note that the feedback term given by ( 3 ) is a special case of $(20)$ where $f\left(e_{n}\right)=\rho e_{n}$. Another choice might be $f\left(e_{n}\right)=e_{n}^{2}$ which yields exponentially stable error dynamics for $\left|e_{0}\right| \leqslant \rho<1$, see (19). This choice in $(20)$ yields

$G\left(x_{n}, y_{n}\right)=\left(4-5 x_{n}-3 y_{n}\right) e_{n}$.

Selection of $f\left(e_{n}\right)=e_{n}^{m}$ where $m>1$ also yields similar results.

This technique could also be applied to other chaotic systems as well. To see this, assume that the systems in (1) and (2) are given as

$x_{n+1}=F\left(x_{n}\right)$,

$y_{n+1}=F\left(y_{n}\right)+G\left(x_{n}, y_{n}\right)$,

where $F: \mathbb{R}^{\prime \prime} \rightarrow \mathbb{R}^{n}$ is an arbitrary map such that (21) exhibits chaotic behaviour, and $G: \mathbb{R}^{n} \times \mathbb{R}^{n} \rightarrow$ $\mathbb{R}^{n}$ is the fecdback term. Let the error be defined as in (4) and assume that for error dynamics given by (19), $e=0$ is asymptotically stable. If we choose the feedback term as

$G\left(x_{n}, y_{n}\right)=F\left(x_{n}\right)-F\left(y_{n}\right)-f\left(e_{n}\right)$,

then the error dynamics will be given by (19), hence the synchronization can be achieved. In particular, if we choose $f(e)=A e$ where $A$ is a $n \times n$ constant matrix whose eigenvalues are in the unit disc (i.e. the magnitudes of the eigenvalues are less than 1 ), then the synchronization is exponential and statements similar to Fact 2 and Fact 3 hold, i.e. the scheme is robust with respect to noise and parameter mismatch. This could easily follow, since in this case there exist constants $M>0$ and $|\rho|<1$ such that $\left\|A^{n}\right\| \leqslant M|\rho|^{n}$ for $n \geqslant 1$, see, e.g., Ref. [20, p. 279]. The rest could be proven by following the steps in Facts 2 and 3.

\section{Conclusions}

In this Letter we proposed a simple feedback scheme for the synchronization of two coupled logisiic maps. We showed that with this scheme the synchronization error decays exponentially to zero. As a result of exponential synchronization, we showed that this scheme is robust with respect to noise and parameter mismatch. We also presented some simulation results and some possible generalizations of this scheme to the synchronization of other chaotic systems.

\section{References}

[1] L.M. Pecora, T.L. Carroll, Phys. Rev. Lett. 64 (1990) 821.

[2] M.d.S. Viera, A.J. Lichtenberg, M.A. Lieberman, Phys. Rev. A 46 (1992) R7359.

[3] H. Fujisaka, T. Yamada, Progress Theor. Phys. 69 (1983) 32.

[4] K. Pyragas, Phys. Lett. A 181 (1993) 203.

[5] K.M. Cuomo, A.V. Oppenheim, Phys. Rev. Lett. 71 (1993) 65.

[6] K.S. Halle, C.W. Wu, M. Itoh, L.O. Chua. Int. J. Bifurcation Chaos 3 ( 1993) 469.

[7] L. Kocarev, K.S. Halle, K. Eckert, L.O. Chua, U. Parlitz, Int. J. Bifurcation Chaos 2 (1992) 709.

[8] L. Kocarev, U. Parlitz, Phys. Rev. Lett. 74 (1995) 5028.

[9] L.M. Pecora, T.L. Carroll, Phys. Rev. A. 44 (1991) 2374.

[10] Ö. Morgül, E. Solak, Phys. Rev. E. 54 (1996) 4803.

[11] K. Pyragas, Phys. Lett. A. 170 (1992) 421.

[12] T. Kailath, Linear Systems (Prentice Hall, Englewood Cliffs, NJ, 1980).

[13] Ö. Morgül, E. Solak, Int. J. Bifurcation Chaos 7 (1997) 1307.

[14] M.K. Ali, Phys. Rev. E. 55 (1997) 4804.

[15] A.S. Pikovsky, P. Grassberger, J. Phys. A 24 (1991) 4587.

[16] L. Gardini, R. Abraham, R.J. Record, D. Fournier-Prunaret, Int. J. Bifurcation Chaos 4 (1994) 145.

[17] P. Celka, Physica D 90 (1996) 235. 
[18] Y. Maistrenko, T. Kapitaniak, Phys. Lett. E 54 (1996) 3285.

[19] T. Kapitaniak, M. Sekieta, M. Ogorzalek, Int. J. Bifurcation Chaos 6 (1996) 211.
[20] M.W. Hirsch, S. Smale, Differential Equations, Dynamical Systems and Linear Algebra, Series Pure and Applied Math., Vol. 60 (Academic Press, New York, 1974). 